\title{
LETTER
}

\section{When ultrasound-guided catheterization is useless: back to landmarks!}

\author{
Raphaël Giraud ${ }^{1,2,3^{*}}$ and Karim Bendjelid ${ }^{1,2,3}$ \\ See related research by Maizel et al., http://ccforum.com/content/18/1/R36
}

A study by Maizel and colleagues [1] in a recent issue of Critical Care shows that a resident skilled at inserting a central venous catheter $(\mathrm{CVC})$ via the ultrasound-guided (UG) technique may face difficulties inserting a CVC via the anatomical landmark (LM) technique. Because several studies have demonstrated significantly increased safety, effectiveness, and efficiency of UG vascular access, as compared with cannulation by anatomical LMs, the UG technique became the more broadly recommended procedure [2-4]. However, as residents are trained only in this technique, they are no longer able to perform the LM technique, even when ultrasound is not available or applicable.

In fact, we recently had a patient with extensive subcutaneous emphysema requiring veno-venous extracorporeal membrane oxygenation. Percutaneous cannula implantation, usually performed under ultrasound guidance, has been inserted using the LM technique. Because of emphysema, no visualization of veins was possible (Figure 1).

Despite international recommendations, it seems to be essential to avoid the UG one-way dogmatic approach and continue to train our young colleagues to insert the CVC via a conventional technique because in certain situations in which ultrasound guidance cannot be used, cannulation remains vital for the patient. Thus, if the clinical situation is favorable (anatomy and coagulation are normal, and the patient is not overweight), young doctors could make a puncture test according to the LM technique. The UG technique would be automatically performed in case of failure of the first and single puncture. In this way, the LM technique will continue to be taught so that all doctors can insert catheters in situations when ultrasound is not usable.

\footnotetext{
* Correspondence: raphael.giraud@hcuge.ch

'Intensive Care Service, Geneva University Hospitals, 4 Rue Gabrielle

Perret-Gentil, CH-1211 Geneva 14, Switzerland

${ }^{2}$ Faculty of Medicine, University of Geneva, Rue Michel Servet 1,

1205 Geneva, Switzerland

Full list of author information is available at the end of the article
}

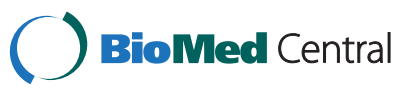

C 2014 Giraud and Bendjelid; licensee BioMed Central Ltd. This is an Open Access article distributed under the terms of the Creative Commons Attribution License (http://creativecommons.org/licenses/by/4.0), which permits unrestricted use, distribution, and reproduction in any medium, provided the original work is properly credited. The Creative Commons Public Domain Dedication waiver (http://creativecommons.org/publicdomain/zero/1.0/) applies to the data made available in this article, unless otherwise stated. 


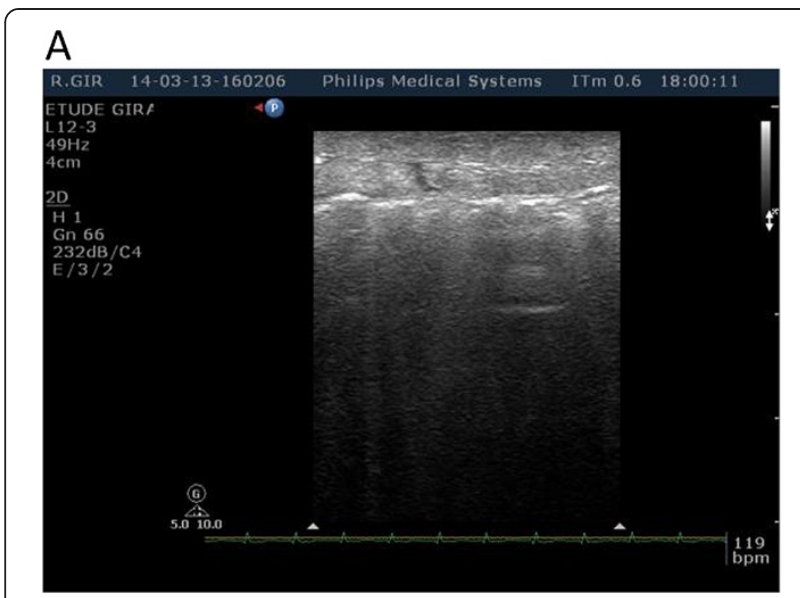

\section{B}

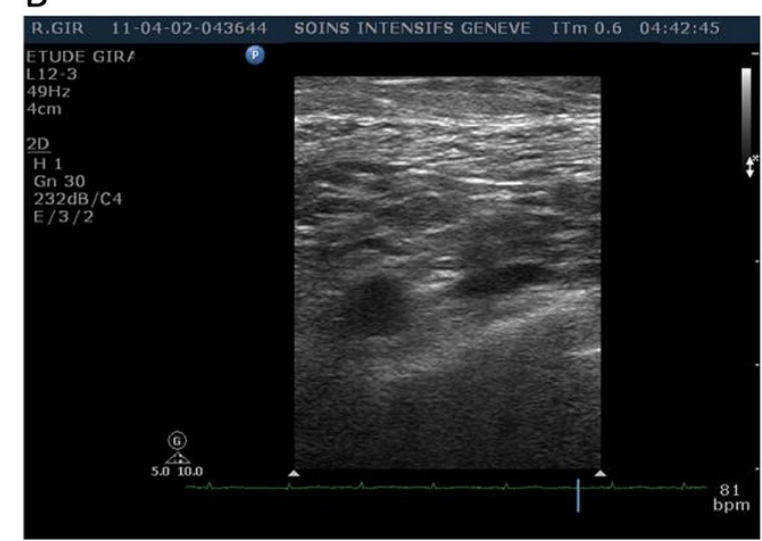

Figure 1 Ultrasound images of the neck for the internal jugular vein location during vascular access puncture (A) with and (B) without subcutaneous emphysema. An impossible ultrasound guidance (A) and a possible ultrasound guidance (B) are shown.

\section{Abbreviations}

CVC: Central venous catheter; LM: Landmark; UG: Ultrasound-guided.

\section{Competing interests}

The authors declare that they have no competing interests.

\section{Author details}

'Intensive Care Service, Geneva University Hospitals, 4 Rue Gabrielle Perret-Gentil, CH-1211 Geneva 14, Switzerland. ${ }^{2}$ Faculty of Medicine, University of Geneva, Rue Michel Servet 1, 1205 Geneva, Switzerland. ${ }^{3}$ Geneva Hemodynamic Research Group, University of Geneva, Rue Michel Servet 1, 1205 Geneva, Switzerland.

\section{Published: 11 Jul 2014}

\section{References}

1. Maizel J, Guyomarc HL, Henon P, Modeliar SS, de Cagny B, Choukroun G, Slama M: Residents learning ultrasound-guided catheterization are not sufficiently skilled to use landmarks. Crit Care 2014, 18:R36.

2. Lamperti M, Bodenham AR, Pittiruti M, Blaivas M, Augoustides JG, Elbarbary M, Pirotte T, Karakitsos D, Ledonne J, Doniger S, Scoppettuolo G, Feller-Kopman D, Schummer W, Biffi R, Desruennes E, Melniker LA, Verghese ST: International evidence-based recommendations on ultrasound-guided vascular access. Intensive Care Med 2012, 38:1105-1117.
3. American Society of Anesthesiologists Task Force on Central Venous Access, Rupp SM, Apfelbaum JL, Blitt C, Caplan RA, Connis RT, Domino KB, Fleisher LA, Grant S, Mark JB, Morray JP, Nickinovich DG, Tung A: Practice guidelines for central venous access: a report by the American Society of Anesthesiologists Task Force on Central Venous Access. Anesthesiology 2012, 116:539-573.

4. Troianos CA, Hartman GS, Glas KE, Skubas NJ, Eberhardt RT, Walker JD Reeves ST, Councils on Intraoperative Echocardiography and Vascular Ultrasound of the American Society of Echocardiography: Guidelines for performing ultrasound guided vascular cannulation: recommendations of the American Society of Echocardiography and the Society of Cardiovascular Anesthesiologists. J Am Soc Echocardiogr 2011, 24:1291-1318.

\section{$10.1186 /$ cc13986}

Cite this article as: Giraud and Bendjelid: When ultrasound-guided catheterization is useless: back to landmarks! Critical Care 2014, 18:452 